\title{
Asociación entre dificultad escolar y recién nacidos de término, pequeños para la edad gestacional
}

School Difficulties in 20-Year-Olds who were born small for gestacional age at term in a regional cohort study. Larroque B, Bertrais S, Czernichow P y col. Pediatrics 2001 uly;vol.108: 111-115 Ann. Intern Med 2000; 133:573-584.

\section{Objetivo}

Investigar la relación entre las dificultades escolares y el haber sido un recién nacido de término pequeño para la edad gestacional (PEG), en adolescentes y adultos jóvenes.

Diseño

Estudio de cohorte regional.

\section{Lugar}

Se utilizaron los registros de maternidad de la ciudad de Haguenau, Francia.

\section{Pacientes}

Se estudiaron un total de 236 recién nacidos de término pequeños para la edad gestacional (PEG) y 281 recién nacidos de término de peso adecuado (PAEG) nacidos entre 1971 y 1978 y fueron evaluados a una edad media de 20,6 $( \pm 2,1)$ años.

Se consideró PEG a los recién nacidos con peso y/o talla debajo del percentilo 3 y los PAEG a los de percentilo 25 a 75 .

\section{Evaluación de factores pronósticos}

Se realizó un análisis de regresión logística* para evaluar el efecto independiente de ser PEG sobre las variables elegidas, ajustando por el efeto de edad materna, nivel educacional materno, nivel socio económico paterno, número de hijos, y sexo.

\section{Medición de resultados principales}

Se estudió:la entrada tardía al secundario (normal a los 11 anos de edad) y la falla para dar o pasar el examen de finalización del secundario (normal a los 18 años de edad).

\section{Resultados principales}

La entrada tardía al secundario fue más frecuente en los niños PEG que en los PAEG (OR: 2.3), luego de ajustar por factores sociales, económicos y culturales. Una significativa mayorproporción de adolescentes PEG fallaron el examen de finalización del secundario que los alumnos PAEG (OR: 1.6).

Se encontró una mayor entrada tardía al secundario en los $P E G$ con perímetro cefálico pequeño que en los $P E G$ con perímetro cefálico adecuado.

\section{Conclusiones}

El nacimiento a término pero pequeño para la edad gestacional se asocia con pobre rendimiento escolar a los 12 y 18 años. La adaptación fetal a las condiciones que provocaron el retardo de crecimiento intrauterino durante la gestación pueden no ser suficientes para mantener un adecuado desarrollo del cerebro.

Fuente de financiamiento:no referida.

\section{Comentario}

El retardo de crecimiento intrauterino (RCIU) está asociado a un aumento en la mortalidad y morbilidad perinatal como asimismo a la falla en el crecimiento postnatal. Diversos estudios se están ocupando de analizar que ocurre con el neurodesarrollo de niños y jóvenes que presentaron RCIU cuyos cerebros se sabe estuvieron expuestos a diferentes factores tóxicos y períodos de disminución de nutrientes y oxigeno durante la vida fetal. El presente trabajo analizó que pasó con niños con y sin RCIU a los 20 años de edad comparando el ingreso y egreso del colegio secundario, encontrando que los niños pequeños para la edad gestacional tuvieron mayor proporción de fallas en la escolaridad que sus pares de peso adecuado.La evaluación se realizó ajustando por factores demográficos y socioculturales involucrados. Es de destacar que este estudio no valoró el coeficiente intelectual de los niños de los dos grupos.

Es importante citar el trabajo realizado por Strauss ${ }^{1}$, quien siguió a los pacientes PEG hasta los 26 años de vida y observó que hubo significativas diferencias en el desempeño académico y profesional con respecto a los PAEG. Sin embargo no se detectaron consecuencias sociales o emocionales a largo plazo en comparación con los PAEG.

La importancia del presente artículo radica en investigar un tema absolutamente controvertido como es el neurodesarrollo en niños nacidos a término con RCIU, en los cuales es muy discutido si tienen problemas de escolaridad. Algunos niegan esta asociación.
Por ejemplo, Sommmerfelt 2 publicó un estudio de niños $\mathrm{PEG}$ seguidos hasta los 5 años con moderado RCIU y no encontró diferencias ; Roth 3 tampoco lo observó en niños seguidos hasta el primer año de vida.

En cambio en los recién nacidos pretérmino, sí está más estudiado y comprobado que tienen fallas en el colegio sobre todo en la infancia temprana y especialmente en la áreas de lecto-escritura y matemáticas 4,5 , siendo esto más frecuente si los prematuros a su vez fueron PEG.

Es importante destacar del estudio de Larroque el seguimiento alejado de los pacientes PEG.Los trabajos citados anteriormente y que no arrojaron diferencias significativas fueron con evaluaciones en los primeros años de vida, y es sabido que las evaluaciones realizadas a muy temprana edad tienen poco valor predictivo en relación a las habilidades futuras de los niños.Su función es determinar si hay un problema de desarrollo o no en un momento determinado para poder intervenir y luego saber si se modifica con el transcurso del tiempo.

Conclusión del comentario: Los hallazgos de este estudio aportan información interesante a la controversia ya que esta basada en datos de seguimiento a largo plazo que ofrece una perspectivas más adecuada del desarrollo de esta particular población.

*Ver glosario

\section{Dra. Veronica Cravedi}

[ Neonatologa de la División de Neonatología del Htal Italiano de Buenos Aires.Docente autorizada de la UBA y Especialista en Desarrollo de recién nacidos prematuros ]

\section{Referencias}

1.Strauss RS.Adult functional outcome of those born small for gestational age: twenty-six-year follow-up of the 1970 British Birth Cohort. JAMA 2000 Feb 2;283(5):625-32. 2.Sommerfelt K, Andersson HW, Sonnander K and col. Behavior in term, small for gestational age preschoolers. Early Hum Dev 2001 dec;65(2):107-21.

3. Roth S, Chang TC, Robson S and col. The neurodevelopmental outcome of term infants with different intrauterine growth characteristics. Early Hum Dev. 1999;55:39-50.

4. Saigal S, Szatmari P, Rosembaum P and col.Intellectual and functional status at school entry of children who weighed 1000 grams or less at birth. J of ped march 1990;116(3):409-416. 5. Sung IK, Vohr B, O W. Growth neurodevelopment outcome of very low birth weight infant with intrauterine growth retardation:comparison with control subjects matched by birth weight and gestational age.J Pediatr 1993 oct;123(4):618-24. 